\title{
Analytical synthesis of function generating spherical four-bar mechanism for the five precision points
}

\author{
Rasim I. Alizade ${ }^{\mathrm{a}, *}$, Özgür Kilit ${ }^{\mathrm{b}}$ \\ a Department of Mechanical Engineering, Izmir Institute of Technology, Urla, Izmir 35437, Turkey \\ b Mechanical Engineering Department, Faculty of Engineering, Ege University, 35040, Bornova, Izmir, Turkey
}

Received 22 October 2002; received in revised form 10 October 2004; accepted 27 December 2004

Available online 22 February 2005

\begin{abstract}
This paper presents an analytical method for synthesis of function generating spherical 4R mechanisms for the five precision points. For the design requirements an additional parameter, reference value of output angle, $\psi_{0}$, was added to angular link length parameters, $\alpha_{i}(i=1, \ldots, 4)$. In the dimensional synthesis procedure, a novel approach of polynomial approximation method was proposed to determine these five design parameters. Using this method, a set of five non-linear equations was easily transformed into a set of fifteen linear equations. Hence, the problem was reduced to the solution of a cubic polynomial equation. Moreover, a graphical method in a CAD environment is proposed to verify the solutions.
\end{abstract}

(C) 2005 Elsevier Ltd. All rights reserved.

\section{Резюме}

Рассматривается пространственный передаточный четырехзвенный механизм с пятью постоянными угловыми параметрами. Сферический механизм 4R предназначен для генерации заданной функции. Для решения нелинейных уравнений кинематического синтеза используется интерполяционный метод приближения функ ций по пяти заданным положениям. Применяя метод суперпозиции для линеаризации нелинейных уравнений получено уравнение четвертой степени. Решение достигается по предложенному четкому методу кинематического синтеза.

(C) 2005 Elsevier Ltd. All rights reserved.

\footnotetext{
${ }^{*}$ Corresponding author. Tel.: +90 232498 6544; fax: +90 2324986505.

E-mail addresses: alizada_rasim@hotmail.com (R.I. Alizade), ozgurkilit@mail.ege.edu.tr (Ö. Kilit).

URLs: http://likya.iyte.edu.tr/mechweb/facultyandstaff/ralizade.htm (R.I. Alizade), http://likya.iyte.edu.tr/mechweb/ facultyandstaff/okilit.htm (Ö. Kilit).
} 
Keywords: Spherical linkage mechanism; Synthesis; Superposition; Non-linear equations; Precision point

\section{Introduction}

Both planar and spherical linkage mechanisms are constrained mechanisms in three-dimensional Euclidean space $\mathbb{R}^{3}$. Their links are constrained to move on planar and spherical surfaces, respectively. Two independent coordinates are necessary and sufficient to describe a point on both planar and spherical surfaces. In this point of view, planar and spherical mechanisms are similar. On the other hand, moving links of both spherical and spatial mechanisms generate three-dimensional movements. That is to say, spherical mechanisms are an intermediate stage between planar and spatial mechanisms [3]. Actually, because of intersecting all of their joint axes at infinity, planar mechanisms are a special case of spherical mechanisms. Similarly, because of intersecting all of their joint axes at the same point, spherical mechanisms are a special case of spatial mechanisms.

Several discussions on the synthesis of function generating spherical mechanisms have been widely studied in [6-8,17,23-25]. Applications of spherical mechanisms to robotic mechanical systems and to rehabilitation treatment of the anatomic joints have been proposed by Chablat and Angeles [5] and Hong et al. [14]. Most industrial manipulators have a spherical wrist, which can be described by the rotations of the end effectors about three revolute joints with intersecting axis. Bruyninckx et al. [2] describes a spherical $4 \mathrm{R}$ wrist, which can be transformed to equivalent spherical 3R wrist by coupled second and third joint axes. The synthesis problem in the design of a function generating spherical four-bar mechanism which describes the input-output motion by using analytical methods of polynomial approximation for given three, four and five precision points were studied by several researchers $[1,9,10,19,20,28]$.

In analytical and optimization synthesis problem, the additional synthesis conditions such as Grashof, assembly, transmission angles, singularity, workspace and others should also be studied. Grashof conditions for spherical 4R linkages were first presented by Freudenstein [11]. Several other studies, $[6,12,13,15,23]$, have been made to derive different forms of spherical rotatability. Kazerounian [15] introduced that the transmission angle formulation in a spherical four-bar linkage is a quartic equation in $t$, tangent of the half of the input angle, and the mobility of the spherical linkage is related to the number of the real roots of this quartic equation. Solutions of this research agree with the ones discussed in other studies [12,22].

The equality and inequality synthesis conditions as gross mobility equations and the conditions of the relative motion for all the links of the $4 \mathrm{R}$ spherical mechanism have been presented by Cervantes-Sanchez et al. [4]. It was also shown in the same study that the $4 \mathrm{R}$ spherical linkage can be classified in 33 types. Ruth and McCarthy [21] introduced 81 spherical linkage types which were separated into three assembly constraints: 27 linkages for $\alpha_{1}+\alpha_{2}+\alpha_{3}+\alpha_{4}<0,27$ linkages for $\alpha_{1}+\alpha_{2}+\alpha_{3}+\alpha_{4}=0$ and 27 linkages for $\alpha_{1}+\alpha_{2}+\alpha_{3}+\alpha_{4}>0$. Here $\alpha$ 's are the angular link-length of spherical four-bar linkage. From these 81 linkages, there are 65 types of folding spherical linkages and 16 non-folding types. Ruth and McCarthy [21] also used the fundamental Burmester's geometrical synthesis theory for describing a CAD (computer aided design) software system for spherical four-bar linkages. The first spherical mechanism computer-aided design program was 
written by Larochelle et al. [16]. Tse and Larochelle also presented a new method for approximating a finite set of $n$ spatial locations with $n$ spherical orientations [26]. The singularity conditions in finite position synthesis of spherical 4R linkage were discussed by McCarthy and Bodduluri [18]. Generalizations of Filemon's construction and Waldron three circle diagram in spherical $4 \mathrm{R}$ synthesis theory gives three quadratic cones and the coupler passes through the specified orientations avoiding singular configurations [18].

As discussed above, some researchers are concerned with additional synthesis conditions as equality and inequality, whereas the others are concerned with analytical synthesis problem and synthesizing the parameters.

The objective of this study was to apply superposition method for linearization of non-linear synthesis equations in the problem of analytical synthesis of function generating spherical $4 \mathrm{R}$ linkage mechanism described by five precision points. Using approximation method and solving of the third order equation, three solutions were found with only one of them being real. Application of these synthesized parameters to spherical $4 \mathrm{R}$ linkage mechanism gives a mechanism that satisfies the given function on the precision points. Also, a constraint condition, minimum deviation area, is represented. It is shown that how the location of the precision points effects the deviation from given function.

\section{Definition of spherical four-bar geometry}

In Fig. 1, vectors of revolute pairs of spherical four-bar mechanism were shown as position vectors and they are named as $\mathbf{A}, \mathbf{B}, \mathbf{C}$ and $\mathbf{D}$, respectively. Vector $\mathbf{A}$ has been aligned to the $x$-axis, vector $\mathbf{B}$ has been put on $x-y$ plane and all vector lengths are assumed as 1 for simplifying the problem. Because the locus of points in space having a given fixed distance from a given point $O$ is called as sphere, all heads of vectors $\mathbf{A}, \mathbf{B}, \mathbf{C}$ and $\mathbf{D}$, are on the surface of the sphere. To derive one simple equation using the terms $\alpha_{1}, \alpha_{2}, \alpha_{3}, \alpha_{4}, \varphi$ and $\psi$, some perpendicular lines to coordinate axes were dropped and formed right-angled triangles.

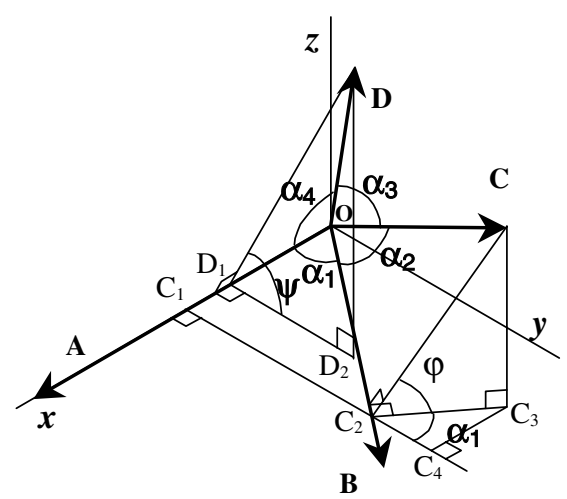

Fig. 1. Position vectors of a spherical four-bar mechanism. 
Using triangular relations following equations can be written:

$$
\left.\begin{array}{llll}
\text { In } \triangle C O C_{2} & \Rightarrow \overline{C C_{2}}=S \alpha_{2} & \text { and } & \overline{O C_{2}}=C \alpha_{2} \\
\text { In } \Delta C C_{2} C_{3} & \Rightarrow \overline{C C_{3}}=S \alpha_{2} S \varphi & \text { and } & \overline{C_{2} C_{3}}=S \alpha_{2} C \varphi \\
\text { In } O C_{1} C_{2} & \Rightarrow \overline{O C_{1}}=C \alpha_{1} C \alpha_{2} & \text { and } & \overline{C_{1} C_{2}}=S \alpha_{1} C \alpha_{2} \\
\text { In } \Delta C_{3} C_{2} C_{4} \Rightarrow \overline{C_{3} C_{4}}=S \alpha_{1} S \alpha_{2} C \varphi & \text { and } & \overline{C_{2} C_{4}}=C \alpha_{1} S \alpha_{2} C \varphi \\
\text { In } \Delta D O D_{1} & \Rightarrow \overline{D D_{1}}=S \alpha_{4} & \text { and } & \overline{O D_{1}}=C \alpha_{4} \\
\text { In } \Delta D D_{1} D_{2} & \Rightarrow \overline{D D_{2}}=S \alpha_{4} S \psi & \text { and } & \overline{D_{1} D_{2}}=S \alpha_{4} C \psi
\end{array}\right\} \text {, }
$$

where $S x$ and $C x$ stand for $\sin (x)$ and $\cos (x)$ respectively. The components of the position vectors $\mathbf{A}, \mathbf{B}, \mathbf{C}$ and $\mathbf{D}$, given in Fig. 1, are calculated by using Eq. (1) as follows:

$$
\begin{aligned}
& \mathbf{A}=\{1,0,0\}^{\mathrm{T}}, \\
& \mathbf{B}=\left\{C \alpha_{1}, S \alpha_{1}, 0\right\}^{\mathrm{T}}, \\
& \mathbf{C}=\left\{C \alpha_{1} C \alpha_{2}-S \alpha_{1} S \alpha_{2} C \varphi, S \alpha_{1} C \alpha_{2}+C \alpha_{1} S \alpha_{2} C \varphi, S \alpha_{2} S \varphi\right\}^{\mathrm{T}}, \\
& \mathbf{D}=\left\{C \alpha_{4}, S \alpha_{4} C \psi, S \alpha_{4} S \psi\right\}^{\mathrm{T}} .
\end{aligned}
$$

By using scalar product of vectors $\mathbf{C}$ and $\mathbf{D}$, the following equation can be written:

$$
C_{x} D_{x}+C_{y} D_{y}+C_{z} D_{z}=C \alpha_{3} .
$$

Substitution of Eq. (2) into Eq. (3) gives a general position equation (or objective function) of spherical four-bar mechanism as follows:

$$
C \alpha_{1} C \alpha_{2} C \alpha_{4}-S \alpha_{1} S \alpha_{2} C \alpha_{4} C \varphi+S \alpha_{1} C \alpha_{2} S \alpha_{4} C \psi+C \alpha_{1} S \alpha_{2} S \alpha_{4} C \varphi C \psi+S \alpha_{2} S \alpha_{4} S \varphi S \psi=C \alpha_{3} .
$$

The problem of finding four constant parameters, $\alpha_{1}, \alpha_{2}, \alpha_{3}$ and $\alpha_{4}$, for a given set of variable input and output angle parameters, $\varphi_{i}$ and $\psi_{i}$, is known as a synthesis problem of spherical fourbar mechanism. Eq. (4) can be transformed into a set of linear equations, Eq. (5), as follows:

$$
P_{1} C \varphi_{i}+P_{2} C \psi_{i}+P_{3} C \varphi_{i} C \psi_{i}+P_{4} S \varphi_{i} S \psi_{i}=1, \quad i=1, \ldots, 4,
$$

where

$$
\begin{aligned}
& P_{1}=-S \alpha_{1} S \alpha_{2} C \alpha_{4} /\left(C \alpha_{3}-C \alpha_{1} C \alpha_{2} C \alpha_{4}\right) \\
& P_{2}=S \alpha_{1} C \alpha_{2} S \alpha_{4} /\left(C \alpha_{3}-C \alpha_{1} C \alpha_{2} C \alpha_{4}\right) \\
& P_{3}=C \alpha_{1} S \alpha_{2} S \alpha_{4} /\left(C \alpha_{3}-C \alpha_{1} C \alpha_{2} C \alpha_{4}\right) \\
& P_{4}=S \alpha_{2} S \alpha_{4} /\left(C \alpha_{3}-C \alpha_{1} C \alpha_{2} C \alpha_{4}\right) .
\end{aligned}
$$

This is a system of linear equations which is a set of four linear equations with four unknowns, $P_{1}$, $P_{2}, P_{3}$, and $P_{4}$. Each precision point corresponds to an equation. After solving the unknowns, angles $\alpha_{1}, \alpha_{2}, \alpha_{3}$ and $\alpha_{4}$ can be easily calculated from Eq. (6).

\section{Synthesis of four-bar mechanism with five parameters}

The main idea in function generating synthesis of spherical four-bar mechanisms is the design of a mechanism so that to provide a prescribed motion between input and output links. Reference angles of input and/or output angles are important in function generation since a definite portion 
of the function has to be satisfied. In this paper, the output angle, $\psi$, was considered as the sum of two angles, reference angle, $\psi_{0}$, and variable angle, $\psi_{i}$. If $\psi_{i}$ is replaced by this sum, $\psi_{0}+\psi_{i}$, the following equation can be obtained:

$$
\begin{aligned}
& C \alpha_{1} C \alpha_{2} C \alpha_{4}-C \alpha_{3}+C \alpha_{1} S \alpha_{2} S \alpha_{4} C \psi_{0} C \varphi_{i} C \psi_{i}-C \alpha_{1} S \alpha_{2} S \alpha_{4} S \psi_{0} C \varphi_{i} S \psi_{i}-S \alpha_{1} S \alpha_{2} C \alpha_{4} C \varphi_{i} \\
& \quad+S \alpha_{1} C \alpha_{2} S \alpha_{4} C \psi_{0} C \psi_{i}-S \alpha_{1} C \alpha_{2} S \alpha_{4} S \psi_{0} S \psi_{i}+S \alpha_{2} S \alpha_{4} S \psi_{0} S \varphi_{i} C \psi_{i}+S \alpha_{2} S \alpha_{4} C \psi_{0} S \varphi_{i} S \psi_{i} \\
& \quad=0, \quad i=1, \ldots, 5 .
\end{aligned}
$$

In this case, we have five unknowns: $\alpha_{1}, \alpha_{2}, \alpha_{3}, \alpha_{4}$ and $\psi_{0}$. Hence the synthesis problem becomes the solution of these unknowns using data set of $\varphi_{i}$ and $\psi_{i}, i=1, \ldots, 5$. The simultaneous equations in Eq. (7) are a set of five non-linear equations with five unknowns.

Division of all terms in Eq. (7) by $C \alpha_{1} S \alpha_{2} S \alpha_{4} C \psi_{0}$ gives the following set of equations,

$$
\begin{aligned}
P_{1} & +P_{2} C \varphi_{i} S \psi_{i}+P_{3} C \varphi_{i}+P_{4} C \psi_{i}+P_{5} S \varphi_{i} S \psi_{i}+P_{6} S \psi_{i}+P_{7} S \varphi_{i} C \psi_{i} \\
& =-C \varphi_{i} C \psi_{i}, \quad i=1, \ldots, 5,
\end{aligned}
$$

where $P_{1}=\left(C \alpha_{1} C \alpha_{2} C \alpha_{4}-C \alpha_{3}\right) /\left(C \alpha_{1} S \alpha_{2} S \alpha_{4} C \psi_{0}\right), P_{2}=-S \psi_{0} / C \psi_{0} ; P_{3}=-\left(S \alpha_{1} C \alpha_{4}\right) /\left(C \alpha_{1} S \alpha_{4} C \psi_{0}\right)$, $P_{4}=\left(S \alpha_{1} C \alpha_{2}\right) /\left(C \alpha_{1} S \alpha_{2}\right), P_{5}=1 / C \alpha_{1}, P_{6}=-\left(S \alpha_{1} C \alpha_{2} S \psi_{0}\right) /\left(C \alpha_{1} S \alpha_{2} \psi_{0}\right)$ and $P P_{7}=S \psi_{0} /\left(C \alpha_{1} C \psi_{0}\right)$.

In this case, there are seven unknowns but only five linear equations. Since the number of linear equations is less than the number of unknowns, Eq. (8) is an Underdetermined Linear System of Equations. To find an analytical solution of Eq. (8), two more equations are required. These two additional equations can be found by making some manipulations and arrangements between constant parameters of this equation as follows:

$$
\left.\begin{array}{l}
P_{6}=P_{2} P_{4} \\
P_{7}=-P_{2} P_{5}
\end{array}\right\} .
$$

If $\lambda_{1}$ and $\lambda_{2}$ are defined as non-linear parameters in place of $P_{6}$ and $P_{7}$, respectively, Eq. (9) becomes

$$
\left.\begin{array}{l}
P_{2} P_{4}-\lambda_{1}=0 \\
P_{2} P_{5}+\lambda_{2}=0
\end{array}\right\} .
$$

The terms with $\lambda_{1}$ and $\lambda_{2}$ can be gathered on the same side of equation

$$
P_{1}+P_{2} C \varphi_{i} S \psi_{i}+P_{3} C \varphi_{i}+P_{4} C \psi_{i}+P_{5} S \varphi_{i} S \psi_{i}=-C \varphi_{i} C \psi_{i}-\lambda_{1} S \psi_{i}-\lambda_{2} S \varphi_{i} C \psi_{i}, \quad i=1, \ldots, 5 .
$$

Since all the terms of the left side of Eq. (11) are linear, it was assumed that the constructional parameters, $P_{k}(k=1, \ldots, 5)$, are linearly proportional with $\lambda_{1}$ and $\lambda_{2}$ as follows:

$$
P_{k}=l_{k}+\lambda_{1} m_{k}+\lambda_{2} n_{k}, \quad k=1, \ldots, 5 .
$$

Substituting Eq. (10) and (12) into Eq. (11) gives

$$
\begin{aligned}
& \lambda_{1}\left(m_{1}+m_{2} C \varphi_{i} S \psi_{i}+m_{3} C \varphi_{i}+m_{4} C \psi_{i}+m_{5} S \varphi_{i} S \psi_{i}\right)+\lambda_{2}\left(n_{1}+n_{2} C \varphi_{i} S \psi_{i}+n_{3} C \varphi_{i}\right. \\
& \left.\quad+n_{4} C \psi_{i}+n_{5} S \varphi_{i} S \psi_{i}\right)+\left(l_{1}+l_{2} C \varphi_{i} S \psi_{i}+l_{3} C \varphi_{i}+l_{4} C \psi_{i}+l_{5} S \varphi_{i} S \psi_{i}\right) \\
& \quad=\lambda_{1}\left(-S \psi_{i}\right)+\lambda_{2}\left(-S \varphi_{i} C \psi_{i}\right)+\left(-C \varphi_{i} C \psi_{i}\right), \quad i=1, \ldots, 5 .
\end{aligned}
$$


The coefficients of the terms with $\lambda_{1}, \lambda_{2}$ and without $\lambda$ in Eq. (13) can be equalized and represented in the standard matrix form as

$$
\mathbf{A}_{\mathbf{m}} \mathbf{x}_{\mathbf{m}}=\mathbf{b}_{\mathbf{m}}
$$

where $\mathbf{A}_{\mathbf{m}}$ is matrix of coefficients, $\mathbf{x}_{\mathbf{m}}$ is the column vector of variables and $\mathbf{b}_{\mathbf{m}}$ is the column vector of solutions. It should be noted here that $\mathbf{A}_{\mathbf{m}}$ is a Block Diagonal Matrix. This kind of matrix is a square diagonal matrix in which the diagonal elements, $[\mathbf{X}]_{5 \times 5}$, are square matrices, and the offdiagonal elements are 0 . Therefore, Eq. (14) can be given explicitly as

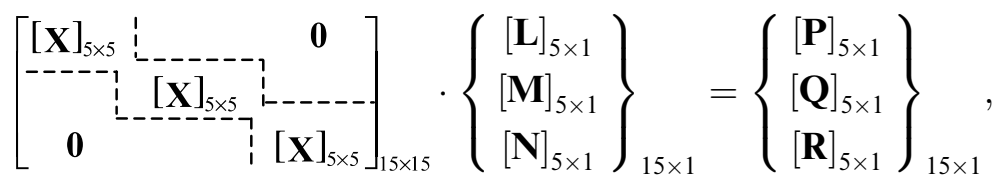

where

$$
\begin{gathered}
\mathbf{X}=\left[\begin{array}{lllll}
1 & C \varphi_{1} S \psi_{1} & C \varphi_{1} & C \psi_{1} & S \varphi_{1} S \psi_{1} \\
1 & C \varphi_{2} S \psi_{2} & C \varphi_{2} & C \psi_{2} & S \varphi_{2} S \psi_{2} \\
\ldots & \ldots & \ldots & \ldots & \ldots \\
1 & C \varphi_{5} S \psi_{5} & C \varphi_{5} & C \psi_{5} & S \varphi_{5} S \psi_{5}
\end{array}\right] ; \quad \mathbf{L}=\left\{\begin{array}{c}
l_{1} \\
l_{2} \\
\cdots \\
l_{5}
\end{array}\right\} ; \quad \mathbf{M}=\left\{\begin{array}{c}
m_{1} \\
m_{2} \\
\cdots \\
m_{5}
\end{array}\right\} ; \\
\mathbf{N}=\left\{\begin{array}{c}
n_{1} \\
n_{2} \\
\cdots \\
n_{5}
\end{array}\right\} ; \quad \mathbf{P}=\left\{\begin{array}{c}
C \varphi_{1} C \psi_{1} \\
C \varphi_{2} C \psi_{2} \\
\ldots \\
C \varphi_{5} C \psi_{5}
\end{array}\right\} ; \quad \mathbf{Q}=\left\{\begin{array}{c}
S \psi_{1} \\
S \psi_{2} \\
S \psi_{5}
\end{array}\right\} \text { and } \mathbf{R}=\left\{\begin{array}{c}
S \varphi_{1} C \psi_{1} \\
S \varphi_{2} C \psi_{2} \\
S \varphi_{5} C \psi_{5}
\end{array}\right\} .
\end{gathered}
$$

To find a solution, $\mathbf{A}_{\mathbf{m}}$ matrix must be non-singular. A square matrix is non-singular if and only if its determinant is non-zero (i.e. $\left|\mathbf{A}_{\mathbf{m}}\right| \neq 0$ ). Solving Eq. (15) gives $l_{k}, m_{k}$ and $n_{k}, k=1, \ldots, 5$ values. Substitution of Eq. (12) into Eq. (10) gives

$$
\left\{\begin{array}{l}
\left(l_{2}+\lambda_{1} m_{2}+\lambda_{2} n_{2}\right)\left(l_{4}+\lambda_{1} m_{4}+\lambda_{2} n_{4}\right)-\lambda_{1}=0 \\
\left(l_{2}+\lambda_{1} m_{2}+\lambda_{2} n_{2}\right)\left(l_{5}+\lambda_{1} m_{5}+\lambda_{2} n_{5}\right)+\lambda_{2}=0
\end{array}\right\} .
$$

Arranging Eq. (16) yields

$$
\left\{\begin{array}{l}
A_{1} \lambda_{1}^{2}+A_{2} \lambda_{2}^{2}+A_{3} \lambda_{1} \lambda_{2}+A_{4} \lambda_{1}+A_{5} \lambda_{2}+A_{6}=0, \\
B_{1} \lambda_{1}^{2}+B_{2} \lambda_{2}^{2}+B_{3} \lambda_{1} \lambda_{2}+B_{4} \lambda_{1}+B_{5} \lambda_{2}+B_{6}=0 .
\end{array}\right\},
$$

where

$$
\begin{aligned}
& A_{1}=m_{2} m_{4} \quad A_{2}=n_{2} n_{4} \quad A_{3}=m_{2} n_{4}+m_{4} n_{2} \quad A_{4}=l_{2} m_{4}+l_{4} m_{2}-1 \quad A_{5}=l_{2} n_{4}+l_{4} n_{2} \\
& A_{6}=l_{2} l_{4}, \\
& B_{1}=m_{2} m_{5} \quad B_{2}=n_{2} n_{5} \quad B_{3}=m_{2} n_{5}+m_{5} n_{2} \quad B_{4}=l_{2} m_{5}+l_{5} m_{2} \quad B_{5}=l_{2} n_{5}+l_{5} n_{2}+1 \\
& B_{6}=l_{2} l_{5} .
\end{aligned}
$$


By multiplying the second line of Eq. (17) with $-A_{2} / B_{2}$ and then by summation of the first and second lines side by side and rearrangement of the terms gives,

$$
\lambda_{2}=\frac{C_{1} \lambda_{1}^{2}+C_{2} \lambda_{1}+C_{3}}{C_{4} \lambda_{1}+C_{5}},
$$

where

$$
\begin{aligned}
& C_{1}=A_{2} B_{1}-A_{1} B_{2} \quad C_{2}=A_{2} B_{4}-A_{4} B_{2} \quad C_{3}=A_{2} B_{6}-A_{6} B_{2} \quad C_{4}=A_{3} B_{2}-A_{2} B_{3} \\
& C_{5}=A_{5} B_{2}-A_{2} B_{5} .
\end{aligned}
$$

Substituting $\lambda_{2}$ and $\lambda_{2}^{2}$ into the first line of Eq. (17) gives a fourth order equation of $\lambda_{1}$

$$
D_{4} \lambda_{1}^{4}+D_{3} \lambda_{1}^{3}+D_{2} \lambda_{1}^{2}+D_{1} \lambda_{1}+D_{0}=0
$$

where $D_{4}=A_{1} C_{4}^{2}+A_{2} C_{1}^{2}+A_{3} C_{1} C_{4}$. When $A_{1}, A_{2}, A_{3}, C_{1}$ and $C_{4}$ constants are put in their places in Eq. (19), it is important to see that $D_{4}$ becomes zero, which reduces the order of the polynomial Eq. (19) from fourth to third degree. So finally, the following third degree of polynomial in $\lambda_{1}$ is found,

$$
D_{3} \lambda_{1}^{3}+D_{2} \lambda_{1}^{2}+D_{1} \lambda_{1}+D_{0}=0
$$

where

$$
\begin{aligned}
& D_{0}=A_{2} C_{3}^{2}+A_{6} C_{5}^{2}+A_{5} C_{3} C_{5}, \\
& D_{1}=2 A_{2} C_{2} C_{3}+A_{3} C_{3} C_{5}+A_{4} C_{5}^{2}+2 A_{6} C_{4} C_{5}+A_{5} C_{2} C_{5}+A_{5} C_{3} C_{4}, \\
& D_{2}=A_{1} C_{5}^{2}+A_{2} C_{2}^{2}+2 A_{2} C_{1} C_{3}+A_{3} C_{2} C_{5}+A_{3} C_{3} C_{4}+2 A_{4} C_{4} C_{5}+A_{6} C_{4}^{2}+A_{5} C_{1} C_{5}+A_{5} C_{2} C_{4}, \\
& D_{3}=2 A_{1} C_{4} C_{5}+2 A_{2} C_{1} C_{2}+A_{3} C_{1} C_{5}+A_{3} C_{2} C_{4}+A_{4} C_{4}^{2}+A_{5} C_{1} C_{4} .
\end{aligned}
$$

To solve this cubic Eq. (20), without loss of generality it may be assumed that the coefficient of $\lambda_{1}^{3}$ can be taken as 1 . To do this, all terms are divided by $D_{3}$ as follows:

$$
\lambda_{1}^{3}+a_{2} \lambda_{1}^{2}+a_{1} \lambda_{1}+a_{0}=0
$$

where $a_{2}=D_{2} / D_{3}, a_{1}=D_{1} / D_{3}$ and $a_{0}=D_{0} / D_{3}$. Weisstein [27] informs that the solution of the general cubic equations was published by Gerolamo Cardano (1501-1576). This method is used here to solve this cubic equation. A substitution of the form

$$
\lambda_{1}=x-\delta
$$

is used to eliminate the $a_{2}$ term. Putting this term into Eq. (21) gives

$$
x^{3}+\left(a_{2}-3 \delta\right) x^{2}+\left(a_{1}-2 a_{2} \delta+3 \delta^{2}\right) x+\left(a_{0}-a_{1} \delta+a_{2} \delta^{2}-\delta^{3}\right)=0 .
$$

The $x^{2}$ is eliminated by lettering $\delta=a_{2} / 3$. So Eq. (23) becomes

$$
x^{3}+p x=q,
$$

where $p=\left(3 a_{1}-a_{2}^{2}\right) / 3$ and $q=\left(9 a_{1} a_{2}-27 a_{0}-2 a_{2}^{3}\right) / 27$. Using Vièta's substitution in Eq. (25)

$$
x=\omega-p /(3 \omega) \text {, }
$$


Eq. (24) becomes the following quadratic equation of $\omega^{3}$ :

$$
\left(\omega^{3}\right)^{2}-q\left(\omega^{3}\right)-\frac{1}{27} p^{3}=0 .
$$

Solution of this quadratic equation gives following Eq. (27):

$$
\omega^{3}=\frac{1}{2} q \pm \sqrt{\frac{1}{4} q^{2}+\frac{1}{27} p^{3}} .
$$

Putting all the relevant terms in place in Eq. (22) gives

$$
\lambda_{1}=\omega-\frac{3 a_{1}-a_{2}^{2}}{9 \omega}-\frac{a_{2}}{3} .
$$

Substituting this value for $\lambda_{1}$ in Eq. (28) into Eq. (18) gives $\lambda_{2}$. $\lambda_{1}$ and $\lambda_{2}$ are used to find $P_{k}$, $k=1, \ldots, 5$ in Eq. (12). Finally, using these $P_{k}$ values all of the design parameters, $\alpha_{1}, \alpha_{2}, \alpha_{3}$, $\alpha_{4}$ and $\psi_{0}$, can be found as in Eq. (29).

$$
\begin{aligned}
& \alpha_{1}=\cos ^{-1}\left(1 / P_{5}\right), \\
& \alpha_{2}=\tan ^{-1}\left(\tan \alpha_{1} / P_{4}\right), \\
& \alpha_{3}=\cos ^{-1}\left(\cos \alpha_{1} \cos \alpha_{2} \cos \alpha_{4}-P_{1} \cos \alpha_{1} \sin \alpha_{2} \sin \alpha_{4} \cos \psi_{0}\right), \\
& \alpha_{4}=\tan ^{-1}\left[-\tan \alpha_{1} /\left(P_{3} \cos \psi_{0}\right)\right], \\
& \psi_{0}=\tan ^{-1}\left(-P_{2}\right) .
\end{aligned}
$$

\section{Scale of given function}

Given function is in the form of $y=f(x)$. In most of the industrial applications, designers may want to use some part and some scale of this given function in their design. For example, a designer may need to design a spherical for-bar linkage mechanism satisfying, say, an exponential function, $y=x^{a}$. A part of this function, $x_{\min } \leqslant x_{i} \leqslant x_{\max }$ should be scaled to the input angle as $\varphi_{\min } \leqslant \varphi_{i} \leqslant \varphi_{\max }$ (where $i$ is the precision number). Also the output angle, $\psi$, can be scaled independently in the range of $\psi_{\min } \leqslant \psi_{i} \leqslant \psi_{\max }$. To scale function $y=f(x)$ to function $\psi=f(\varphi)$ linearly, following equations should be satisfied.

$$
\frac{\varphi-\varphi_{\min }}{x-x_{\min }}=\frac{\varphi_{\max }-\varphi_{\min }}{x_{\max }-x_{\min }} ; \quad \frac{\psi-\psi_{\min }}{y-y_{\min }}=\frac{\psi_{\max }-\psi_{\min }}{y_{\max }-y_{\min }} .
$$

Deriving $x$ and $y$ values from Eq. (30) and substituting to the given function gives following $\psi=f(\varphi)$ function. Similarly, any continuous $y=f(x)$ function can be scaled to $\psi=f(\varphi)$ function for given range.

$$
\begin{aligned}
\psi= & \left(\frac{\psi_{\max }-\psi_{\min }}{y_{\max }-y_{\min }}\right)\left[\varphi\left(\frac{x_{\max }-x_{\min }}{\varphi_{\max }-\varphi_{\min }}\right)-\varphi_{\min }\left(\frac{x_{\max }-x_{\min }}{\varphi_{\max }-\varphi_{\min }}\right)+x_{\min }\right]^{a} \\
& -y_{\min }\left(\frac{\psi_{\max }-\psi_{\min }}{y_{\max }-y_{\min }}\right)+\psi_{\min }
\end{aligned}
$$




\section{Constraint conditions}

As seen in section 3, calculating the constructional parameters is straightforward for a given set of precision points. The designer will only needs to determine the input angles, $\varphi_{i}(i=1, \ldots, 5)$. Then, output angles, $\psi_{i}(i=1, \ldots, 5)$, will be calculated by using scaled form of given function. Actually infinite number of set of precision points satisfying the given function can be selected. Every set of precision points leads related set of constructional parameters. That is to say, another set of precision points means another completely different mechanism. So, additional constraint conditions are required to select better mechanism according to the designer's requirements.

Many constraint conditions have been given by some researchers before $[4,6,11-13,15,23]$. In most of the applications, the input crank in a linkage should be able to rotate $360^{\circ}$. McCarthy [17] gave the spherical version of Grashof's criterion as $s+l<p+q$. Where $s+l$ is the summation of the angular link-lengths of shortest and longest links while $p+q$ is the summation of the reminders. Another constraint is transmission angle. Transmission angle, $\zeta$, is the outer angle between coupler and driven links. Drive force that is transmitted by the coupler link acts upon driven link by this angle. Longitudinal component of this force creates a reaction force at revolute joint while the lateral component acts as a working force. So, $\cos (\zeta)$ is used comprehensively as a measurement of the efficiency of linkages. In some other design problems, angular link-length ratios of the spherical mechanism should be in a specific range. All of these constraint conditions have been discussed comprehensively before. In this paper we represented a constraint condition: MDA (minimum deviation area), to select the mechanism that has a minimum amount of deviation from the given function.

Synthesized mechanism will exactly pass through the precision points only. Whereas, out of the precision points there will always be some amount of positive or negative deviation (or error) as seen in Fig. 2. As seen in Fig. 3, by assuming different set of precision points the profile of the resulting function and total area of the deviation from the given function is changed. In some high precision industries, this deviation may be highly risky. A number of constraints can be defined to

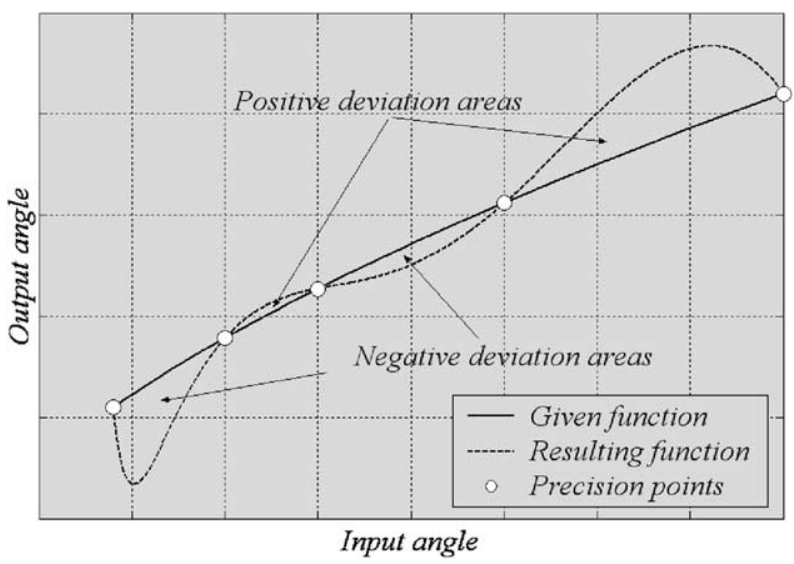

Fig. 2. Comparison of given (desired) and resulting function of $\psi$. 


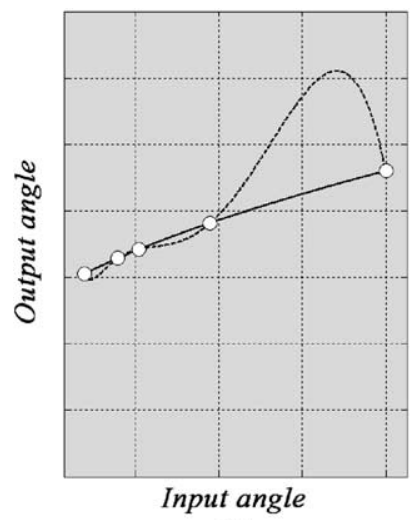

(a)

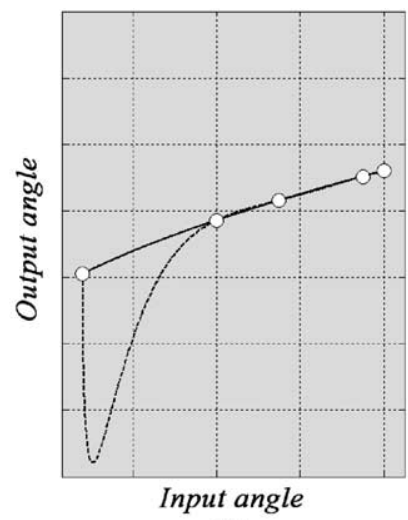

(b)

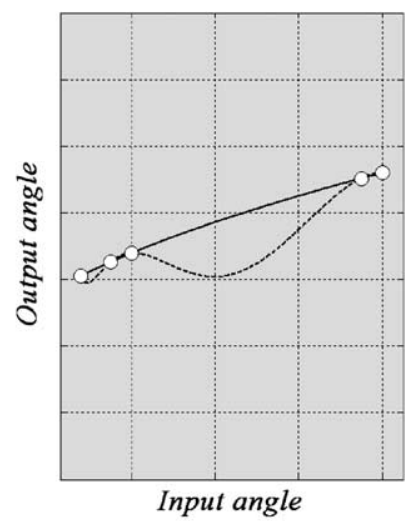

(c)

Fig. 3. Effect of the location of the precision points to deviation. Precision points are collected near to (a) first point, (b) last point and (c) both first and last points (solid lines are given, dashed lines are resulting functions).

control this deviation (e.g. minimum positive deviation, minimum negative deviation etc.). Using MDA constraint, all of the precision points should be selected so that the absolute value of the total deviation area will be the minimum.

\section{Algorithm of the design procedure}

In this section, an algorithm was developed to apply all of these equations and criteria to the synthesis problem. Although satisfying the given function, not all sets of the precision points can lead to a working spherical four-bar linkage. Two solutions of quadratic Eq. (26) may be both real, or both complex. If roots are complex, this means that this mechanism cannot be exist. Therefore, the roots of this quadratic equation must be checked to be sure that the solution sets are formed by only the real roots. $\psi_{i}$ angles can be calculated by using $\psi=f(\varphi)$ equation for given $\varphi_{i}$ angles. Because $\varphi_{\min }$ and $\varphi_{\max }$ are the limits of the input angle, these precision points have been fixed. The only variable parameters were assumed as $\varphi_{2}, \varphi_{3}$ and $\varphi_{4}$. If any two of the $\varphi$ values are assumed as same, this will cause to be the same of two rows of $\mathbf{X}$ matrix in Eq. (15). In this case, the rank of the $\mathbf{X}$ matrix will be decreased from five to four and no solution will be reached. Because of this situation, every precision point should be taken as different. Input angle was divided into $n_{d}$ (number of division) parts. So input angle increments, $\delta$, were calculated as $\left(\varphi_{\max }-\varphi_{\min }\right) /$ $n_{d}$. So, $\varphi_{2}$ was changed from $\varphi_{1}+\delta$ to $\varphi_{5}-3 * \delta, \varphi_{3}$ was changed from $\varphi_{2}+\delta$ to $\varphi_{5}-2 * \delta$ and $\varphi_{4}$ was changed from $\varphi_{3}+\delta$ to $\varphi_{5}-\delta$. Possible number for every variable input angles, $n$, can be calculated as following Eq. (32):

$$
n=n_{\mathrm{d}}-n_{\mathrm{v}},
$$

where $n_{\mathrm{d}}$ is division number and $n_{\mathrm{v}}$ is variable number. $n_{v}$ was taken three in this paper. Because precision points will not be repeated, total number of the selection sets, $S$, can be calculated as follows: 


$$
S=\sum_{i=1}^{n} i(n+1-i)=n \sum_{i=1}^{n} i+\sum_{i=1}^{n} i-\sum_{i=1}^{n} i^{2} .
$$

Plugging $\sum_{i=1}^{n} i=n(n+1) / 2$ and $\sum_{i=1}^{n} i^{2}=n\left(2 n^{2}+3 n+1\right) / 6$ terms into Eq. (33) yields the following Eq. (34):

$$
S=n\left(n^{2}+3 n+2\right) / 6 \text {. }
$$

Exponential changing of the number of the set was shown in Fig. 5. At the end of the design procedure, not only the constant parameters of this linkage mechanism but also the precision points themselves have to be documented. To know the location of the precision points, at which this mechanism exactly passes through, may be highly important for designers. The flowchart of the developed synthesis algorithm is presented in Fig. 4. It should be noted here that the only applied constraint was MDA in this algorithm. A designer can also use as many criteria as he

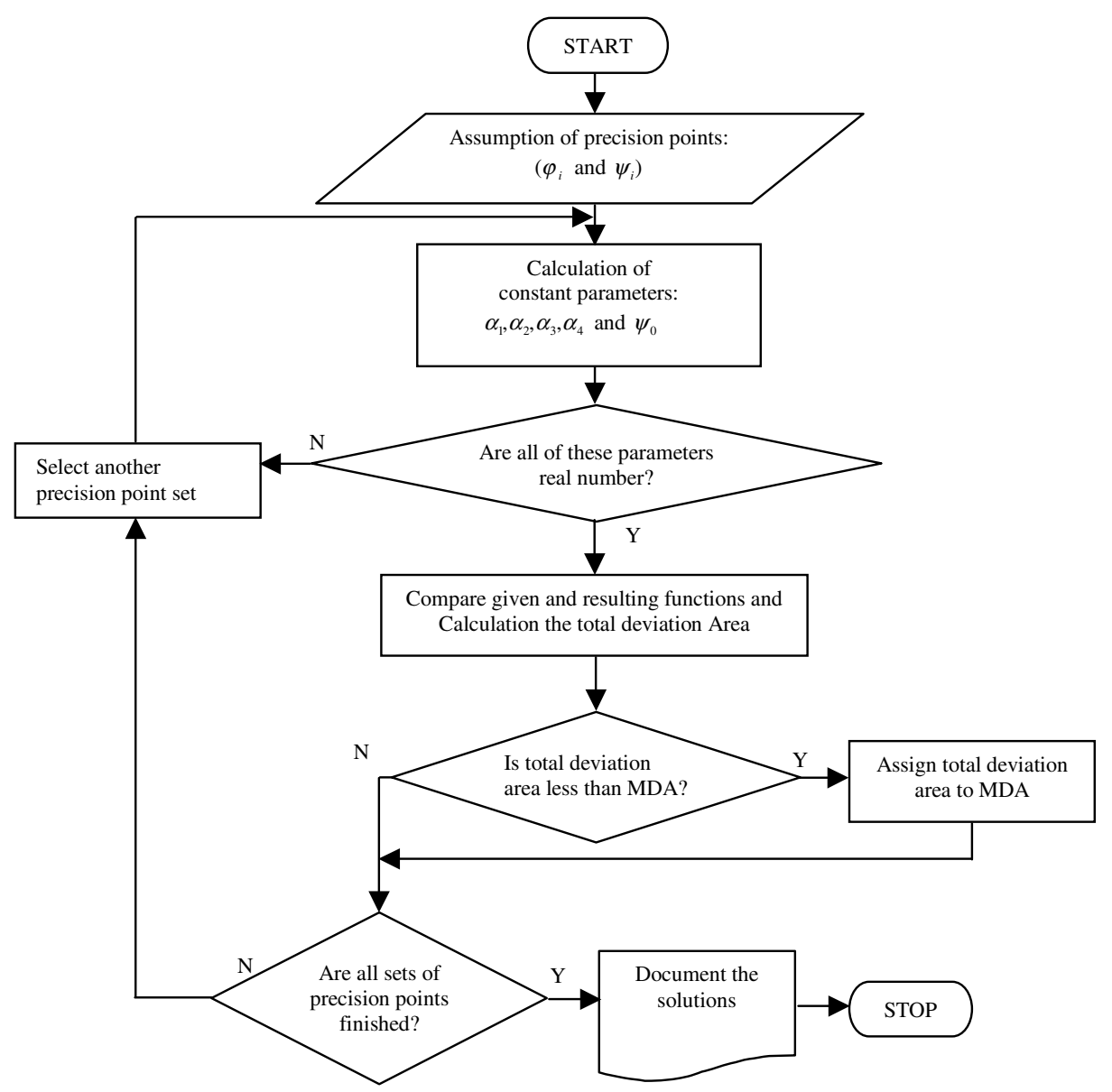

Fig. 4. Flowchart for synthesis algorithm. 


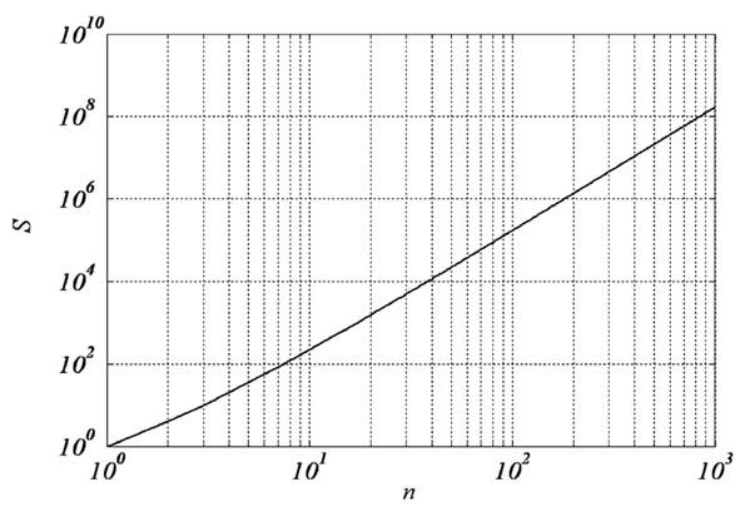

Fig. 5. Exponential function of $S$, total number of selection sets relative to $n$, number of the steps for every variable parameters.

required. Every criterion, as MDA, transmission angle, angular link-length ratios, etc. can be attached to this algorithm easily. Intersection of these conditions gives a solution set of synthesized linkages satisfying all of these conditions simultaneously.

\section{Numerical example}

In this example, a function generating synthesis of a spherical four-bar linkage was attempted. The given function was selected as $y=x^{0.6}$. Input and output angles, $\varphi_{i}$ and $\psi_{i}$, are thought as a scale of $x$ and $y$, respectively. Limits were given as $1 \leqslant x \leqslant 5 ; 1^{0.6} \leqslant y_{i} \leqslant 5^{0.6} ; 8^{\circ} \leqslant \varphi \leqslant 80^{\circ}$ and $5^{\circ} \leqslant \psi \leqslant 160^{\circ}$. In this paper $\delta$ was taken as 1 . A P4-2 GHz computer system was used to run the algorithm in Fig. 4. Total CPU time was found as $8.25 \mathrm{~s}$. Solutions were shown in Table 1 and Fig. 6. CAD drawing of the synthesized mechanism was shown in Fig. 7. It is important to say that, after complex link-length solutions have been excluded, some of the remainders were found to be negative. Actually when we try to verify these solutions by using geometrical method that was given in Appendix A, it is seen that these linkages also satisfy the given function. It is because negative signed $\alpha_{2}$, say, at a $\varphi$ input angle is the same thing with the positive signed of the same $\alpha_{2}$ at a $\varphi+\pi$ input angle. But because negative signed link-length was not defined, only positive signed ones were excepted, though they are possible geometrically.

Table 1

(Panel A) Input and output parameters and (Panel B) constant parameters of the linkage mechanism in which the MDA value is reached

\begin{tabular}{llllll}
\hline$i$ & 1 & 2 & 3 & 4 & 5 \\
\hline Panel A & & & & & \\
$\phi_{i}\left({ }^{\circ}\right)$ & 8.00000 & 18.00000 & 37.00000 & 59.00000 & 80.00000 \\
$\psi_{i}\left({ }^{\circ}\right)$ & 5.00000 & 33.92784 & 79.20331 & 123.11566 & 160.00000 \\
Panel B & & & & \\
$\alpha_{1}\left({ }^{\circ}\right)$ & $\alpha_{2}\left({ }^{\circ}\right)$ & $\alpha_{3}\left({ }^{\circ}\right)$ & $\alpha_{4}\left({ }^{\circ}\right)$ & $\psi_{0}\left({ }^{\circ}\right)$ & $\operatorname{MDA}\left({ }^{\circ 2}\right)$ \\
39.37419 & 89.66027 & 94.44498 & 34.26372 & 11.02554 & 8.55170 \\
\hline
\end{tabular}



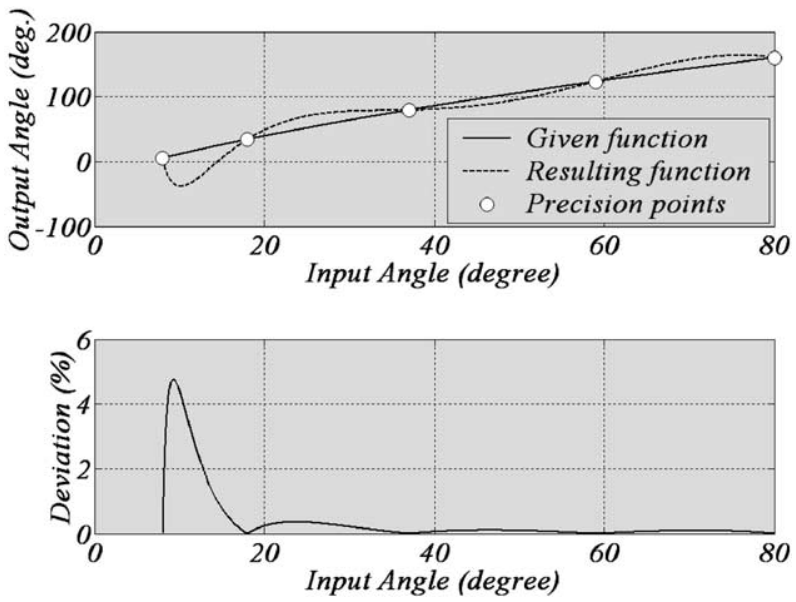

Fig. 6. Changing of output angle and deviation by changing the input angle.

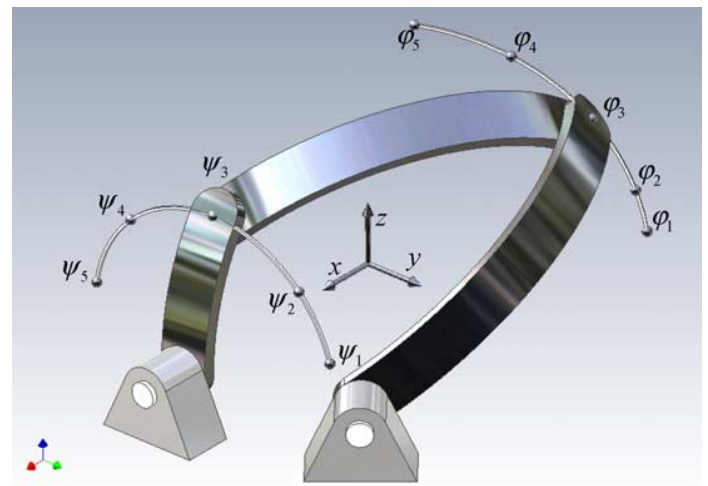

Fig. 7. CAD drawing of the synthesized mechanism.

\section{Conclusions}

In this paper, a detailed geometrical derivation of the general motion equation of a spherical four-bar linkage was given with an additional parameter, reference of the output angle, in a readily intelligible manner. By using a novel polynomial approximation method, a set of five non-linear equations was transformed into a set of 15 linear equations. Further, a constraint condition (MDA: minimum deviation area) is proposed as a criteria to choose the most precise linkage among all of the solution sets. Furthermore, in function generation synthesis procedure, it was observed that the location of the precision points had a dramatical effect on this constraint. It is concluded that the MDA has been reached in a state of approximately equispaced distribution of precision points. 


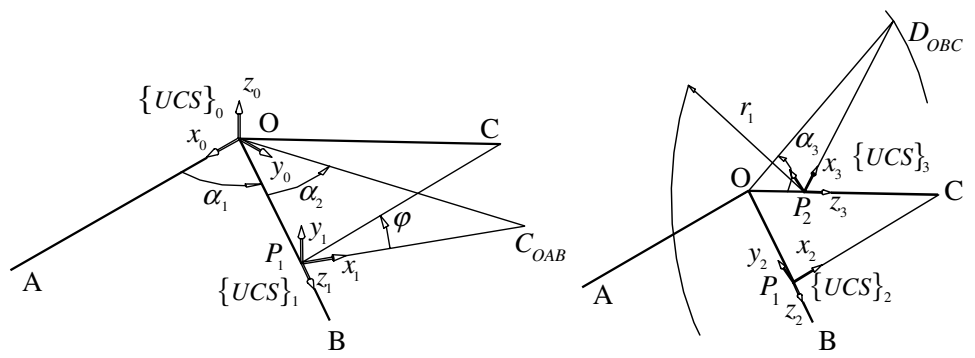

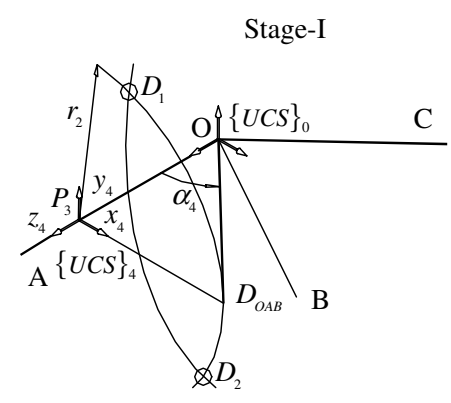

Stage-III

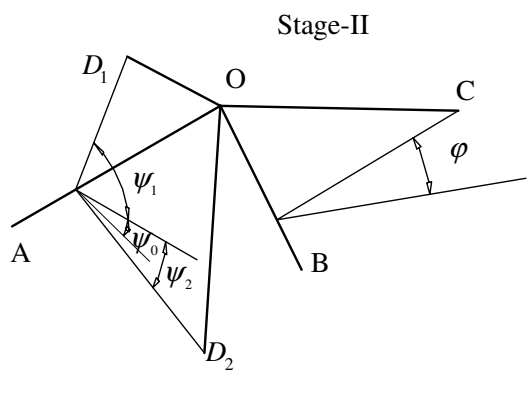

Stage-IV

Fig. 8. Geometrical verification procedure.

\section{Appendix A. Verification of the results with CAD tools}

Once all constructional parameters are known, output angles, $\psi_{i}$, can be checked for given input angles, $\varphi_{i}$, geometrically, by using CAD programs. In this paper AutoCAD 2000 program was used for this verification purposes. Following command procedure was shown geometrically in Fig. 8.

\begin{tabular}{|c|c|c|c|}
\hline Stage & Command & Explanation & Result \\
\hline Stage-I & $\begin{array}{l}<\text { line }> \\
<\text { rotate }> \\
<\text { rotate }> \\
<\text { UCS }> \\
<\text { rotate }>\end{array}$ & $\begin{array}{l}\text { From }<0,0,0>\text { to }<1,0,0> \\
\text { Select } O A \text { line and rotate } \alpha_{1} \text { degrees. } \\
\text { Select } O B \text { line and rotate } \alpha_{2} \text { degrees. } \\
\text { Align } z \text {-axis to } O B \text { line } \\
\text { Select } O C_{O A B} \text { line and rotate } \varphi \text { degrees }\end{array}$ & $\begin{array}{l}O A \text { line } \\
O B \text { line } \\
O C_{O A B} \text { line } \\
\{U C S\}_{1} \\
O C \text { line }\end{array}$ \\
\hline Stage-II & $\begin{array}{l}<\mathrm{UCS}> \\
<\text { rotate }> \\
<\text { line }> \\
<\mathrm{UCS}> \\
<\text { circle }>\end{array}$ & $\begin{array}{l}\text { Align } x \text {-axis to } P_{1} C \text { and } y \text {-axis to } P_{1} O \text {. } \\
\text { Select } O C \text {, rotate } \alpha_{3} \text { degrees } \\
\text { From } D_{\mathrm{OBC}} \text { to perpendicular to } O C \\
\text { Locate the origin at } P_{2} \text {, align } z \text {-axis to } O C \\
\text { Center point is } P_{2} \text {, radius is } P_{2} D_{O B C} \text { line }\end{array}$ & $\begin{array}{l}\{U C S\}_{2} \\
O D_{O B C} \text { line } \\
P_{2} \text { point } \\
\{U C S\}_{3} \\
\text { First circle }\end{array}$ \\
\hline Stage-III & $\begin{array}{l}<\mathrm{UCS}> \\
<\text { rotate }> \\
<\text { line }> \\
<\mathrm{UCS}> \\
<\text { circle }>\end{array}$ & $\begin{array}{l}\text { Align UCS to WCS (World Coordinate System) } \\
\text { Select } O A \text { line and rotate } \alpha_{4} \text { degrees } \\
\text { From } D_{O A B} \text { point to perpendicular to } O A \\
\text { Locate the origin at } P_{3} \text { point, align } z \text {-axis to } O A \text { line } \\
\text { Center point is } P_{3} \text {, radius is } P_{3} D_{O A B} \text { line }\end{array}$ & $\begin{array}{l}\{U C S\}_{0} \\
O D_{O A B} \text { line } \\
P_{3} \text { point } \\
\{U C S\}_{4} \\
\text { Second circle }\end{array}$ \\
\hline
\end{tabular}


Appendix A (continued)

\begin{tabular}{llll}
\hline Stage-IV & $\begin{array}{l}<\text { line }> \\
<\text { rotate } \\
<\text { dimension }>\end{array}$ & $\begin{array}{l}\text { From origin to the two intersection points of circles } \\
\text { Select } P_{3} D_{O A B} \text { line, rotate } \psi_{0} \text { degrees } \\
\text { Dimension } O D_{1} \text { and } O D_{2} \text { lines from reference line }\end{array}$ & $\begin{array}{l}O D_{1} \text { and } O D_{2} \text { lines } \\
\text { Reference line } \\
\text { Two solutions } \\
\text { of the output angle }\end{array}$ \\
\end{tabular}

At the end of Stage-II and Stage-III, two circles (base circles of two cones) were drawn. There are three possibilities for two cones that are sharing the same apex point in 3D space:

(a) Not intersecting cones which corresponds to "no solution".

(b) Tangent cones which corresponds to "one solution".

(c) Intersecting cones which corresponds to "two solutions".

If these cones are intersecting, only one of these two solutions satisfies the given function.

\section{References}

[1] R.I. Alizade, Synthesis of four-bar spherical mechanism on five parameters, J. Mech. Eng., Russian Academy of Science (ANR) 6 (1994) (in Russian).

[2] H. Bruyninckx, H. Thielemans, J.D. Schutter, Efficient kinematics of a spherical 4R wrist by means of an equivalent 3R wrist, IFToMM, Mech. Mach. Theory 33 (6) (1998) 649-659.

[3] A. Castellet, F. Thomas, Characterization of the self-motion set of the orthogonal spherical mechanism, Mech. Mach. Theory 34 (1999) 59-88.

[4] J.J. Cervantes-Sanchez, H.I. Medellin-Castillo, A robust classification scheme for spherical 4R linkages, Mech. Mach. Theory 37 (2002) 1145-1163.

[5] D. Chablat, J. Angeles, The computation of all 4R serial spherical wrists with an isotropic architecture, ASME J. Mech. Design 125 (2003) 275-280.

[6] C.H. Chiang, Kinematics of Spherical Mechanisms, Cambridge University Press, Cambridge, 1988.

[7] V.V. Dobrovolskii, Theory of the spherical mechanisms, Machgiz (1947) 231 (in Russian).

[8] H.J. Dowler, J. Duffy, D. Tesar, A generalized study of four and five multiply separated positions in spherical kinematics, Mech. Mach. Theory. 13 (1978) 409-436.

[9] K. Farhang, A. Midha, A.K. Bajaj, Synthesis of harmonic motion generation linkages Part-I, Function generation, ASME, J. Mech. Transm. Automat. Design 110 (1) (1988) 16-21.

[10] K. Farhang, Y.S. Zargar, Design of spherical 4R mechanisms: function generation for the entire motion cycle, ASME, J. Mech. Design 121 (1999) 521-528.

[11] F. Freudenstein, On the determination of the type of spherical four-link mechanisms, Contemporary problems in the TMM, AS of the USSR (1965) 193-196.

[12] K.C. Gupta, Rotatability considerations for spherical four-bar linkages with applications to robot wrist design, J. Mech. Transm. Automat. Design (1986) 387-391.

[13] K.C. Gupta, A.S. Beloiu, Branch and circuit defect elimination in spherical four-bar linkages, Mech. Mach. Theory. 33 (5) (1998) 491-504.

[14] B. Hong, A. Erdman, K. Ham, Design of adjustable spherical four-bar linkages as continuous passive motion devices for anatomic joints rehabilitation, 96-DETC/MECH-1220, 1996.

[15] K. Kazerounian, R. Solecki, Mobility analysis of general bi-modal four-bar linkages based on their transmission angle, Mech. Mach. Theory 28 (3) (1993) 437-445.

[16] P. Larochelle, J.R. Dooley, A. Murray, J.M. McCarthy, SPHINX - Software for synthesizing spherical 4R mechanisms, Proceedings of the 1993 NSF Design and Manufacturing Systems Conference, University of North Carolina at Charlotte, vol. 1, 1993, pp. 607-611. 
[17] J.M. McCarthy, Geometric Design of Linkages, Springer, Berlin, 2000.

[18] J.M. McCarthy, R.M. Bodduluri, Avoiding singular configurations in finite position synthesis of spherical $4 \mathrm{R}$ linkages, Mech. Mach. Theory 35 (2000) 457-462.

[19] A.V. Mohan Rao, G.N. Sandor, D. Kohli, A.H. Soni, Closed form synthesis of spatial function generating mechanism for the maximum number of precision points, J. Eng. Industry 95 (1973) 725-736.

[20] A.P. Murray, J.M. McCarthy, A linkage map for spherical four position synthesis, ASME Tech. Conf. Boston. MA, 1995, pp. 833-844.

[21] D.A. Ruth, J.M. McCarthy, The design of spherical 4R linkages for four specified orientations, Mech. Mach. Theory 34 (1999) 677-692.

[22] A.H. Soni, Unique description of all spherical four-bar linkages, Discussion, ASME J. Eng. Industry 6 (3) (1970) $563-566$.

[23] A.H. Soni, L. Harrisberger, The design of spherical drag-link mechanism, ASME, J. Eng. Industry 89 (1967) 171181.

[24] C.H. Suh, C.W. Redcliffe, Kinematics and Mechanisms Design, Wiley, NY, 1978.

[25] D.S. Takhvelidzeh, N.S. Davitashvili, Theoretical basis design of plane and spherical linkage mechanisms, Tbilisi. (1975) 206 (in Russian).

[26] D.M. Tse, P.M. Larochelle, Approximating spatial locations with spherical orientations for spherical mechanism design, ASME, J. Mech. Design 122 (2000) 457-463.

[27] E.W. Weisstein, Cubic Equation From MathWorld-A Wolfram Web Resource. Available from: http:// mathworld.wolfram.com/CubicEquation.html.

[28] J.R. Zimmerman, Four-precision synthesis of the spherical four-bar function generator, Mech. Mach. Theory 2 (1967) 133-139. 